\title{
Acomodação familiar e criticismo percebido em pacientes com transtorno obsessivo-compulsivo
}

\author{
Family accommodation and perceived criticism in \\ patients with obsessive-compulsive disorder \\ Ygor Arzeno Ferrão', Marcelo dos Santos Florão²
}

\section{RESUMO}

Objetivo: $O$ transtorno obsessivo-compulsivo (TOC) causa importante impacto sobre a dinâmica familiar. Frequentemente, ocorrem modificações na rotina da família, a qual acaba se adaptando aos sintomas e às exigências do paciente, fenômeno denominado acomodação familiar. Portadores de TOC podem sentir-se, ainda, alvo de críticas por parte de pessoas de seu convívio. Alguns estudos associam pior prognóstico a maiores Índices de Acomodação Familiar e criticismo percebido. Este artigo tem como objetivo verificar possíveis associações entre tipos de sintomas predominantes (dimensões) determinados pela escala DYBOCS, em relação ao funcionamento familiar e percepção crítica dos pacientes. Método: Quarenta e nove pacientes e seus familiares foram avaliados por meio da aplicação de escalas para medição dos índices de criticismo percebido (Perceived Criticism Scale) e acomodação familiar (Family Accommodation Scale), além de escalas para medir a intensidade dos sintomas obsessivo-compulsivos (Dimensional Yale-Brown Obsessive-Compulsive Scale, Yale-Brown ObsessiveCompulsive Scale), depressivos e ansiosos (Hamilton and Beck depression and anxiety inventories). Compararam-se os resultados aos índices obtidos pela DYBOCS e a outras variáveis clínicas relacionadas ao TOC. Resultados: Encontraram-se correlações estatísticas entre gravidade das dimensões agressividade e contaminação, com maior acomodação familiar. Foi verificado também maior criticismo por parte dos pacientes portadores de colecionismo. Conclusão: Diferentes subtipos de TOC exercem diferentes padrões de influência no contexto familiar. Mais estudos com esse enfoque são necessários para melhor orientação comportamental a pacientes e familiares.

\begin{abstract}
Objective: The obsessive-compulsive disorder causes significant impact on the family function, leading to changes in the routine of the family, which adapt itself according to the symptoms and the requirements of the patient, a family phenomenon known as accommodation. OCD patients may be still subject to criticism by those of its relationships. Some studies evince worse prognosis related to higher rates of family accommodation and perceived criticism. This paper aims to de-
\end{abstract}

1 Universidade Federal de Ciências da Saúde de Porto Alegre (UFCSPA), Centro Universitário Metodista/IPA, Serviço de Psiquiatria do Hospital Materno Infantil Presidente Vargas, Consórcio Brasileiro de Pesquisa em Transtornos do Espectro Obsessivo-Compulsivo. 2 Hospital Psiquiátrico São Pedro.

$3 / 2 / 2010$

Endereço para correspondência: Ygor Arzeno Ferrão Rua Padre Chagas, 185/403 - 90570-080 - Porto Alegre, RS

E-mail:ygoraf@terra.com.br 


\section{Keywords}

Obsessive compulsive

disorder, family

accommodation, perceived

criticism. termine possible associations between types of predominant symptoms (dimensions determined by the scale DYBOCS) and the family accommodation and critical perception of the patients. Method: Forty-nine patients and their relatives were evaluated by the Perceived Criticism Scale, Family Accommodation Scale, the Dimensional Yale-Brown Obsessive-Compulsive Scale, the Yale-Brown Obsessive-Compulsive Scale and the Hamilton and Beck depression and anxiety scales. Results: There was significant statistical correlation between severities of the aggressive and contamination/ cleaning dimensions with higher family accommodation. There was also a higher perceived criticism for patients with hoarding. Conclusion: Different contents of obsessive-compulsive symptoms perform different patterns of influence in the family context. These results remain more speculation and studies with proper designs and sample size must be conducted.

\section{INTRODUÇÃO}

O transtorno obsessivo-compulsivo (TOC) abrange um amplo espectro de sintomas que representam múltiplos domínios psicopatológicos, incluindo percepções, cognições, emoções, relacionamentos sociais e diversos comportamentos motores'. As obsessões são definidas como ideias, pensamentos, impulsos ou imagens que são vivenciados como intrusivos e inadequados e causam acentuada ansiedade ou sofrimento; enquanto as compulsões são comportamentos repetitivos ou atos mentais cujo objetivo é prevenir ou reduzir a ansiedade ou sofrimento, em vez de oferecer prazer ou gratificação; geralmente ou são excessivas ou não têm conexão realista com aquilo que visam neutralizar ou evitar². Os conteúdos das obsessões são vários, por exemplo: obsessões de contaminação, dúvidas repetidas, necessidade de organização, impulsos agressivos ou horrorizantes e imagens sexuais. Exemplos de compulsões são: lavagem ou limpeza, verificação, contagem, colecionismo, simetria, entre outros ${ }^{2-4}$.

Os sintomas do TOC têm forte impacto sobre a dinâmica de familiares e cuidadores ${ }^{5-7}$. Muitas vezes os membros da família acabam se ajustando aos sintomas e às exigências do paciente e até mesmo apoiam a realização dos rituais e dos comportamentos compulsivos, envolvidos em um progressivo processo de acomodação familiar. Compreendendo o fenômeno da acomodação familiar, pode-se entender o grau de desgaste e estresse presentes na família ${ }^{8}$. A inserção da família como fator mantenedor ou ativador dos sintomas no TOC parece estar de acordo com teorias cognitivas, principalmente no que diz respeito ao reforço negativo dos sintomas. A participação da família no TOC se distribui entre aquela que apoia e participa plenamente dos comportamentos (polo de acomodação) até aquela que se recusa a participar e se opõe ao paciente (polo antagonístico). Há ainda um terceiro padrão, em que a família se encontra dividida, com membros no polo de acomodação e outros membros no antagonístico?.

Além disso, o portador de TOC pode muitas vezes sentirse alvo de críticas, expressas ou não, por parte de pessoas de seu convívio. Tais percepções são usualmente mensura- das em escala específica que fornece o índice de criticismo percebido. Da mesma maneira que na acomodação familiar, Steketee et al. ${ }^{10}$ relacionaram altos índices de criticismo e hostilidade percebidos pelo paciente em relação aos seus familiares com um pior desempenho terapêutico, especialmente na terapia comportamental ${ }^{10}$. Por outro lado, Guedes $^{8}$ encontrou uma correlação positiva entre a gravidade do TOC e a acomodação familiar ${ }^{8}$. De modo interessante, encontrou também que pacientes com rituais de limpeza eram mais frequentes em famílias com alto índice de acomodação, enquanto as famílias de pacientes com rituais de verificação tinham índice de acomodação moderado ou leve. Dessa forma, poder-se-ia pressupor que os conteúdos obsessivos e os tipos de compulsões, bem como a intensidade desses sintomas, poderiam produzir maior ou menor grau de acomodação familiar. Isso só se tornou possível com a recente abordagem dimensional do TOC, em que vários estudos de análise fatorial demonstraram seis dimensões de sintomas obsessivo-compulsivos como sendo as mais frequentes: agressividade; contaminação e limpeza; simetria e exatidão; colecionismo; sexual-religiosa; e diversos ${ }^{1,11}$. Esses estudos resultaram na elaboração de um instrumento que permitiu avaliar cada uma das dimensões de sintomas obsessivo-compulsivos (SOC), a Dimensional Yale-Brown Obsessive-Compulsive Scale (DYBOCS) ${ }^{12}$.

Assim, o presente estudo tem como objetivo realizar uma análise dos níveis de acomodação familiar e criticismo percebidos de acordo com as dimensões de SOC da DYBOCS, verificando a influência de fatores de confusão, como intensidade dos SOC e de sintomas depressivos e ansiosos, duração e curso do TOC, juízo crítico e número de comorbidades.

\section{MÉTODOS}

Este estudo transversal avaliou clinicamente 49 pacientes ambulatoriais com TOC, de acordo com o DSM-IV, e seus respectivos familiares ou cônjuges $(n=47)$, quanto à acomodação familiar e ao criticismo percebido. Os pacientes foram selecionados consecutivamente, a partir de três serviços de 
referência em TOC: Projeto de Transtorno Obsessivo-Compulsivo (PROTOC) da Faculdade de Medicina da Universidade de São Paulo, Hospital das Clínicas de São Paulo (USP) $(n=7)$; Ambulatório de Transtornos do Espectro ObsessivoCompulsivo (ATEOC) do Serviço de Psiquiatria do Hospital Materno Infantil Presidente Vargas $(n=36)$; Ambulatório de Transtornos de Ansiedade do Hospital de Clínicas de Porto Alegre $(n=6)$. Essa amostra é proveniente de outro estudo que avaliou pacientes com TOC quanto a fatores de risco para resposta aos tratamentos convencionais ${ }^{13}$.

Um paciente não concordou em participar e outro foi excluído da amostra por ter realizado capsulotomia por termocoagulação. Todos os pacientes assinaram um Termo de Consentimento Livre e Esclarecido, aprovado pelos comitês de ética das instituições envolvidas no estudo inicial, respeitando a Declaração de Helsinki e a Resolução no 196, de 1996, do Conselho Nacional de Saúde. A descrição de características sociodemográficas e clínicas da amostra está na tabela 1.

Tabela 1. Características sociodemográficas e clínicas da amostra de pacientes

\begin{tabular}{|c|c|c|}
\hline Características & $\mathrm{n}$ & $\%$ \\
\hline Gênero masculino & 22 & 45 \\
\hline $\begin{array}{l}\text { Estado civil } \\
\text { (sem cônjuge) }\end{array}$ & 20 & 41 \\
\hline $\begin{array}{l}\text { Ocupação profissional } \\
\text { (desempregado) }\end{array}$ & 22 & 45 \\
\hline \multicolumn{3}{|l|}{ Classe socioeconômica* } \\
\hline A & 3 & 6,1 \\
\hline B & 18 & 36,7 \\
\hline C & 25 & 51,0 \\
\hline D & 3 & 6,1 \\
\hline \multicolumn{3}{|c|}{ Familiares entrevistados $(\mathrm{n}=47)$} \\
\hline Mãe & 20 & 42,6 \\
\hline Pai & 2 & 4,3 \\
\hline Filho(a) & 1 & 2,1 \\
\hline Irmão(ã) & 8 & 17,0 \\
\hline \multirow[t]{2}{*}{ Cônjuge } & 16 & 34,0 \\
\hline & Média & Desvio-padrão \\
\hline Idade & 38,5 & 11,5 \\
\hline Número de anos de estudo & 12,0 & 4,2 \\
\hline $\begin{array}{l}\text { Número de comorbidades } \\
\text { psiquiátricas de eixo I }\end{array}$ & 2,1 & 1,2 \\
\hline \multicolumn{3}{|l|}{ Intensidade do TOC } \\
\hline YBOCS total & 22,40 & 8,7 \\
\hline YBOCS obsessões & 11,04 & 4,5 \\
\hline YBOCS compulsões & 11,40 & 4,9 \\
\hline DYBOCS & 17,96 & 5,6 \\
\hline \multicolumn{3}{|l|}{$\begin{array}{l}\text { Intensidade de sintomas } \\
\text { depressivos }\end{array}$} \\
\hline HAMD & 9,9 & 5,5 \\
\hline BDI & 18,2 & 10,7 \\
\hline \multicolumn{3}{|c|}{ Intensidade de sintomas ansiosos } \\
\hline HAMA & 10,4 & 5,9 \\
\hline BAI & 16,3 & 10,3 \\
\hline BABS & 7,04 & 4,8 \\
\hline
\end{tabular}

* Classe socioeconômica de acordo com a escala da Associação Brasileira de Instituições de Pesquisa de Mercado (Abipeme); BABS: Brown Assessment of Beliefs Scale; BAI: Beck Anxiety Inventory; BDI: Beck Depression Inventory; HAMA: Escala de Ansiedade de Hamilton; HAMD: Escala de Depressão de Hamilton; DYBOCS: Dimensional YaleBrown Obsessive-Compulsive Scale; YBOCS: Yale-Brown Obsessive-Compulsive Scale.
Todos os 47 pacientes já haviam utilizado medicações com ação predominantemente serotoninérgica (inibidores da recaptação da serotonina - IRS) em algum momento de sua doença. Do total, 35 (74,5\%) usaram antipsicóticos; 25 (55,3\%) pacientes utilizaram estabilizadores do humor; $21(44,7 \%)$ haviam utilizado antidepressivos tricíclicos; e 20 $(42,6 \%)$ fizeram uso de benzodiazepínicos. Quarenta e dois pacientes $(89,4 \%)$ já haviam realizado ou tentado realizar terapia cognitivo-comportamental. Cinco pacientes (10,6\%) já haviam realizado eletroconvulsoterapia. Vinte e um pacientes $(44,7 \%)$ já haviam se internado em serviços de psiquiatria por causa do TOC. Em relação à resposta aos tratamentos convencionais acima, $22(46,8 \%)$ pacientes foram considerados refratários e 25 (53,2\%) foram considerados respondedores.

As principais comorbidades psiquiátricas de eixo I foram: transtornos depressivos ( $n=34,72,3 \%)$; transtornos de ansiedade ( $n=21,44,7 \%)$; dependência química (álcool e canabis) $(n=9,19,1 \%)$ e transtornos de controle de impulsos $(n=8$; 17,0\%). Em relação ao eixo II, o principal cluster encontrado como comórbido foi o C (evitativo, dependente e anancásti$\mathrm{co})(\mathrm{n}=18,38,3 \%)$.

\section{Instrumentos}

\section{Aplicados aos pacientes}

Entrevista Clínica Estruturada para Transtornos de Eixo I do DSM-IV, versão clínica (SCID-I): essa entrevista estruturada encontra-se de acordo com os critérios diagnósticos do DSMIV, da American Psychiatric Association. Constitui-se de seis capítulos que incluem Transtornos do Humor e episódios de Alteração de Humor, Transtornos Psicóticos e Sintomas Associados, Diagnósticos Diferenciais de Transtornos Psicóticos, Transtornos decorrentes do uso e Abuso de Álcool e outras Substâncias e Transtornos de Ansiedade e um capítulo adicional para Transtornos de Controle de Impulsos. Cada capítulo possui perguntas específicas sobre os sintomas de cada transtorno a serem feitas aos pacientes, sendo as respostas transcritas para um caderno de respostas individual de cada paciente. As respostas dadas pelos pacientes são "sim" ou "não"14.

Escala Dimensional de Sintomas Obsessivo-Compulsivos de Yale-Brown (DYBOCS): escala elaborada por James Leckman e cols., seguindo trabalhos anteriores que utilizaram análises fatoriais para agrupar pacientes com determinados tipos de sintomas obsessivo-compulsivos, nas chamadas "dimensões". Assim, obtiveram-se seis dimensões com os seguintes conteúdos: 1) agressividade; 2) sexual e religiosa; 3) simetria, ordenação e arranjo; 4) contaminação e limpeza; 5) colecionismo e 6) diversos. De modo diferente da YBOCS, os escores não são dados para obsessões e compulsões, e sim pela dimensão. Cada dimensão pode possuir obsessões e compulsões relacionadas. As características avaliadas para cada dimensão são o tempo despendido, o desconforto e a incapacitação por aquela dimensão proporcionada, varian- 
do de "0" a "5". Ainda, para se ter o escore total, avalia-se o nível de prejuízo geral do paciente por causa do TOC (varia de "0" a "12"). A escala em si é aplicada pelo entrevistador, mas a lista de sintomas é autoaplicável, sendo apenas conferida pelo entrevistador. Foi traduzida e revisada pela equipe do PROTOC/IPq/FMUSP/USP'2.

Escala de Criticismo Percebido: essa escala possui cinco itens que se referem à percepção que o paciente tem em relação ao familiar mais importante, e esses mesmos cinco itens são respondidos também em relação ao segundo mais importante familiar para o paciente. O instrumento é autoaplicado e cada pergunta é respondida por pontuação por meio de escala analógica visual. As cinco perguntas questionam: 1) o quanto o familiar dá ao paciente suporte emocional; 2) o quanto o paciente considera que o familiar o critica; 3) o quanto o paciente se considera crítico dos familiares; 4) quão triste o paciente fica quando recebe crítica do familiar e 5) o quão efetivo é o familiar em ajudar o paciente nos problemas relativos ao $\mathrm{TOC}^{10}$.

Escala de Sintomas Obsessivo-Compulsivos de Yale-Brown (YBOCS): essa escala foi desenvolvida por Goodman e cols., para resolver o problema de medir a severidade dos sintomas obsessivo-compulsivos, independentemente do conteúdo das obsessões ou compulsões. É aplicada pelo médico ou psiquiatra e se constitui de 10 questões, cada uma avaliada de "0" (sem sintomas) a "4" (severidade extrema das compulsões ou obsessões). Cinco questões dizem respeito a obsessões e cinco, a compulsões. Foi traduzida para o português pelo Programa de Distúrbio Obsessivo-Compulsivo do Departamento de Psiquiatria e Psicologia da Escola Paulista de Medicina e pelo Ambulatório de Ansiedade do Instituto de Psiquiatria da Faculdade de Medicina da Universidade de São Paulo (Fernando Asbahr, Francisco Lotufo Neto, José Alberto Del Porto, Gentil Valentim, e outros) ${ }^{3,4}$.

Escala de Avaliação de Crenças de Brown (BABS): foi desenvolvida por Katharine Phillips, do Butler Hospital, da Brown University School of Medicine, para medir a convicção e o insight que os pacientes têm de suas crenças. Essas crenças incluem delírios e crenças que fazem parte dos pensamentos obsessivos e das fobias. Assim, a escala avalia sete dimensões como: convicção, percepção dos outros sobre as crenças, explicação das diferenças de visão sobre as crenças, ideação fixa, tentativas de confrontar as crenças, insight e ideias/delírios de referência. Cada dimensão pode variar de 0 a 4 (do menos para o mais severo). Encontra-se em fase de tradução e retrotradução ${ }^{15}$.

Escalas de Beck para Depressão (BDI) e Ansiedade (BAl): amplamente utilizadas na prática clínica, foram desenvolvidas por Aaron Beck e cols. em 1961 e 1988, respectivamente. Constam delas escalas categóricas com respostas de " 0 " a "3". De ambas constam 21 questões e elas possuem escores independentes elaborados pela soma dos resultados em cada item ${ }^{16,17}$.
Escala de Hamilton para Depressão (HAMD): foi desenvolvida para quantificar a gravidade da doença depressiva, além de evidenciar alterações dessa condição clínica. Consta dela uma lista de 21 sintomas que devem ser assinalados por ordem de gravidade (de 0 a 4 ou de 0 a 2), baseando-se em uma entrevista. A escala depende da habilidade e experiência do entrevistador, sendo aconselhável a presença de dois entrevistadores, não se aceitando discrepâncias maiores do que um entre avaliadores. Tem sido amplamente utilizada na clínica psiquiátrica e já se encontra traduzida e validada para o português ${ }^{18}$.

Escala de Classificação de Ansiedade de Hamilton (HAMA): avalia o grau de ansiedade e documenta as mudanças desse sintoma. É planejada para ser aplicada pelo médico e para obter respostas objetivas do paciente. Constam dela 14 itens que devem ser pontuados de 0 a 4, conforme a gravidade dos sintomas. Tem sido amplamente utilizada na clínica psiquiátrica e já se encontra traduzida e validada para o português ${ }^{19}$.

Apostila de Primeiro Atendimento do PROTOC: Elaborada pela equipe do PROTOC/FMUSP para abordar aspectos sociodemográficos como: dados de identificação pessoais, histórico escolar e profissional, história médica geral, tratamento psiquiátrico, história familiar e heredograma, classificação socioeconômica, questionário de lateralidade.

\section{Aplicados aos familiares}

Escala de Acomodação Familiar: elaborada por Calvocoressi e cols. em 1995 e traduzida e adaptada por Maria Luísa Guedes, da USP. Constam dela 16 questões com respostas categóricas sobre a influência da doença, modificações na organização e desgaste da família do paciente ${ }^{20}$.

Apostila de Primeiro Atendimento do PROTOC: para confirmação da história familiar, heredograma e fatores epigenéticos.

Pelo menos dois psiquiatras, conjuntamente, realizaram as entrevistas diagnósticas, avaliaram os pacientes e entrevistaram os familiares. Todos os entrevistadores foram devidamente treinados na aplicação dos instrumentos acima citados.

\section{Análise estatística}

A análise estatística foi realizada inicialmente pelo cálculo das frequências e distribuições de cada variável em cada grupo para posterior comparação. Todas as variáveis foram testadas quanto à sua normalidade e homogeneidade de variâncias. Utilizou-se o teste $t$ de Student (variáveis com distribuição normal) ou teste $U$ de Mann-Whitney (distribuição não normal) para comparação das médias e o coeficiente de Pearson (variáveis com distribuição normal) ou de Spearman (distribuição não normal) para avaliar correlações entre variáveis. O nível de significância escolhido foi de 5\%. Adotou-se um valor de $p<0,10$ para indicar tendência à significância estatística. Utilizou-se o Statistical Package for the Social Sciences (SPSS) 15.0. 


\section{RESULTADOS}

A tabela 2 apresenta a relação entre a presença ou ausência de cada dimensão avaliada pela DYBOCS com as respectivas médias nos escores da escala de acomodação familiar: Índice de Acomodação Familiar, Índice de Desgaste Familiar e Consequência para o paciente caso o familiar não realizasse o que ele queria. Obtive-se tendência estatística ( $p=0,094$ ) para maiores índices de consequências ao paciente em virtude da recusa do familiar em participar dos SOC no grupo de pacientes com conteúdo de contaminação/limpeza, quando comparados aos pacientes que não possuíam essa dimensão de sintomas.

Foram realizadas também comparações entre as médias de cada um dos itens da Escala de Criticismo Percebido, de acordo com a presença ou ausência de cada dimensão da DYBOCS. Essas médias foram obtidas para o familiar mais importante (FM) e para o segundo familiar mais importante (fm) para o paciente. A tabela 3 evidencia que os pacientes com SOC de conteúdo agressivo consideram que os familiares menos importantes nos seus cuidados dão maior suporte emocional quando comparados aos familiares de pacientes sem conteúdo agressivo. Consideram também que os familiares de maior importância são mais efetivos em ajudar com os problemas relativos ao TOC do que os familiares de pacientes sem essa dimensão de sintomas. Observou-se uma tendência estatística dos portadores da dimensão colecionismo a possuírem maior crítica em relação aos familiares menos importantes em seus cuidados, quando comparados aos pacientes sem colecionismo. Além disso, esses pacientes relataram maior intensidade de tristeza ao serem criticados por esses mesmos familiares. As demais dimensões não apresentaram resultados significantes em relação ao criticismo percebido.

Correlacionaram-se os escores de cada dimensão da DYBOCS e os escores da Escala de Criticismo Percebido para os FM e fm, bem como os escores dos índices da Escala de Acomodação Familiar (Tabela 4).

A intensidade dos sintomas de conteúdo agressivo medido pela DYBOCS mostrou correlação direta moderada com o Índice de Acomodação Familiar (IAF) e com o Índice de consequências ao paciente devido à recusa do familiar em participar dos SOC.

Já a intensidade dos sintomas de contaminação e limpeza mostrou correlação inversa moderada com a intensidade do suporte emocional prestado pelo fm. Além disso, mostrou correlação direta moderada com: a intensidade da percepção de críticas recebidas pelo FM; a intensidade da tristeza quando criticado pelo FM; o Índice de Acomodação Familiar; o Índice de Desgaste Familiar (IDF); o Índice de consequências ao paciente devido a recusa do familiar em participar dos SOC.

A intensidade dos sintomas de colecionismo evidenciou apenas tendências estatísticas a estar correlacionada inversamente ao suporte emocional recebido pelo FM e pela efetividade em ajudar com problemas relativos ao TOC do fm.

Tabela 2. Comparação entre as médias dos índices de acomodação familiar em relação à presença ou à ausência de diferentes dimensões do TOC

\begin{tabular}{|c|c|c|c|c|c|c|}
\hline & \multicolumn{2}{|c|}{ IAF } & \multicolumn{2}{|c|}{ IDF } & \multicolumn{2}{|c|}{ Conseq. Pac. } \\
\hline & Média (DP) & $U(p)$ & Média (DP) & $U(p)$ & Média (DP) & Up \\
\hline \multicolumn{7}{|l|}{ Dimensão agressividade } \\
\hline Presente $(n=31)$ & $12,80(9,45)$ & 236,5 & $2,22(2,59)$ & 233,0 & $3,80(2,62)$ & 200,5 \\
\hline Ausente $(n=16)$ & $12,62(10,77)$ & $(0,796)$ & $2,06(1,34)$ & $(0,731)$ & $4,87(3,42)$ & 0,283 \\
\hline \multicolumn{7}{|c|}{ Dimensão sexual/religiosa } \\
\hline Presente $(n=23)$ & $13,47(7,70)$ & 219,5 & $2,47(2,87)$ & 254,5 & $4,13(3,10)$ & 264,5 \\
\hline Ausente $(n=24)$ & $12,04(11,59)$ & $(0,228)$ & $1,87(1,36)$ & $(0,641)$ & $4,20(2,81)$ & $(0,805)$ \\
\hline \multicolumn{7}{|c|}{ Dimensão simetria/ordem } \\
\hline Presente $(n=35)$ & $13,48(9,40)$ & 165,5 & $2,42(2,44)$ & 149,5 & $4,57(3,10)$ & 155,0 \\
\hline Ausente $(n=12)$ & $10,58(11,04)$ & $(0,277)$ & $1,41(1,24)$ & $(0,132)$ & $3,00(2,00)$ & $(0,176)$ \\
\hline \multicolumn{7}{|c|}{ Dimensão contaminação/limpeza } \\
\hline Presente $(n=33)$ & $14,06(9,94)$ & 169,5 & $2,36(2,47)$ & 194,5 & $4,69(3,12)$ & 159,5 \\
\hline Ausente $(n=14)$ & $9,64(9,07)$ & $(0,152)$ & $1,71(1,48)$ & $(0,386)$ & $2,92(1,97)$ & $(0,094)^{*}$ \\
\hline \multicolumn{7}{|l|}{ Dimensão colecionismo } \\
\hline Presente $(n=14)$ & $13,00(6,56)$ & 198,0 & $2,00(1,30)$ & 225,0 & $4,64(2,79)$ & 192,0 \\
\hline Ausente $(n=33)$ & $12,63(10,98)$ & $(0,442)$ & $2,24(2,53)$ & $(0,887)$ & $3,96(3,00)$ & $(0,361)$ \\
\hline
\end{tabular}


Tabela 3. Comparação entre as médias dos índices de Criticismo Percebido quanto à presença ou ausência de diferentes dimensões do TOC

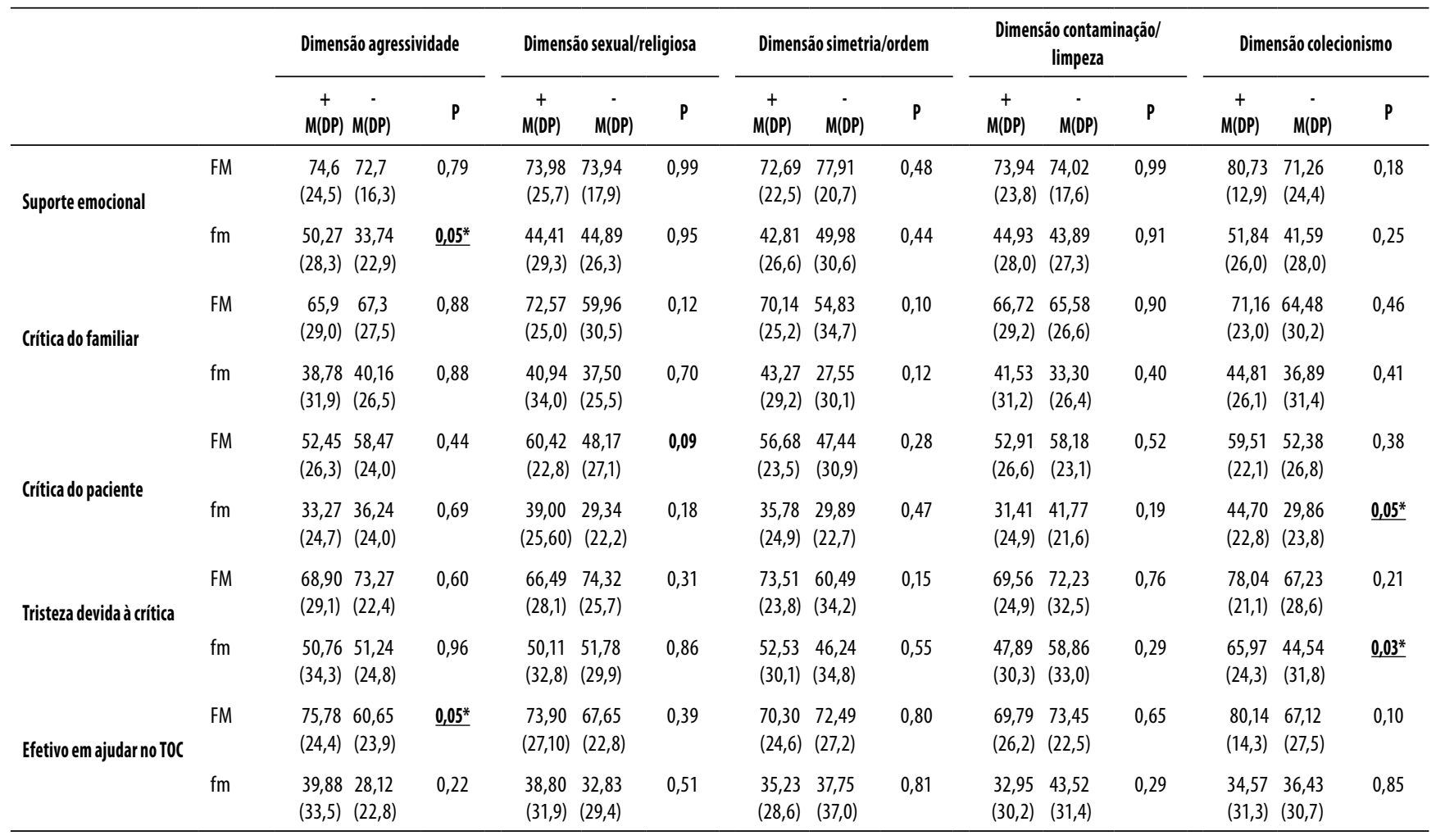

+: presença da dimensão; -: ausência da dimensão; FM: familiar mais importante para o paciente; fm: segundo familiar mais importante para o paciente.

* Significativo a 5\% de probabilidade pelo teste $t$ de Student.

Tabela 4. Correlações entre escores da DYBOCS e medidas de criticismo percebido e acomodação familiar

\begin{tabular}{|c|c|c|c|c|c|c|c|c|c|c|c|c|c|c|c|}
\hline & \multicolumn{3}{|c|}{ Dimensão agressividade } & \multicolumn{3}{|c|}{ Dimensão sexual/religiosa } & \multicolumn{3}{|c|}{ Dimensão simetria/ordem } & \multicolumn{3}{|c|}{ Dimensão contaminação/limpeza } & \multicolumn{3}{|c|}{$\begin{array}{l}\text { Dimensão } \\
\text { colecionismo }\end{array}$} \\
\hline & $n$ & $r$ & $p$ & $n$ & $r$ & $p$ & $\mathrm{n}$ & $r$ & $p$ & $n$ & $r$ & $p$ & $n$ & $r$ & $p$ \\
\hline \multicolumn{16}{|c|}{ Suporte emocional } \\
\hline FM & 33 & 0,122 & 0,500 & 26 & $-0,162$ & 0,428 & 37 & 0,067 & 0,695 & 35 & 0,092 & 0,600 & 15 & $-0,499$ & 0,059 \\
\hline $\mathrm{Fm}$ & 31 & $-0,005$ & 0,978 & 25 & $-0,093$ & 0,658 & 35 & $-0,283$ & 0,099 & 34 & $-0,372$ & $\underline{0,030^{*}}$ & 15 & $-0,356$ & 0,192 \\
\hline \multicolumn{16}{|c|}{ Crítica do familiar } \\
\hline FM & 33 & $-0,177$ & 0,326 & 26 & $-0,143$ & 0,487 & 37 & $-0,006$ & 0,972 & 35 & 0,355 & $0,036^{*}$ & 15 & $-0,011$ & 0,970 \\
\hline $\mathrm{Fm}$ & 31 & $-0,059$ & 0,751 & 25 & 0,328 & 0,109 & 35 & $-0,108$ & 0,536 & 34 & 0,198 & 0,261 & 15 & $-0,082$ & 0,771 \\
\hline \multicolumn{16}{|c|}{ Crítica do paciente } \\
\hline FM & 33 & $-0,192$ & 0,573 & 26 & 0,176 & 0,390 & 37 & $-0,030$ & 0,860 & 35 & 0,075 & 0,668 & 15 & 0,045 & 0,873 \\
\hline $\mathrm{Fm}$ & 31 & $-0,072$ & 0,699 & 25 & 0,074 & 0,724 & 35 & $-0,249$ & 0,149 & 34 & $-0,006$ & 0,972 & 15 & 0,004 & 0,990 \\
\hline \multicolumn{16}{|c|}{ Tristeza devida à crítica } \\
\hline FM & 33 & 0,203 & 0,257 & 26 & 0,106 & 0,606 & 37 & 0,151 & 0,373 & 35 & 0,337 & $\underline{0,048^{*}}$ & 15 & 0,424 & 0,115 \\
\hline $\mathrm{Fm}$ & 31 & 0,087 & 0,641 & 25 & 0,250 & 0,227 & 35 & 0,115 & 0,510 & 34 & 0,044 & 0,807 & 15 & 0,459 & 0,086 \\
\hline \multicolumn{16}{|c|}{ Efetivo em ajudar no TOC } \\
\hline FM & 33 & $-0,033$ & 0,857 & 26 & $-0,222$ & 0,276 & 37 & $-0,026$ & 0,878 & 35 & $-0,097$ & 0,581 & 15 & $-0,361$ & 0,187 \\
\hline $\mathrm{fm}$ & 31 & 0,109 & 0,558 & 25 & $-0,058$ & 0,783 & 35 & $-0,072$ & 0,682 & 34 & $-0,138$ & 0,436 & 15 & $-0,479$ & 0,071 \\
\hline $\mathrm{AF}$ & 31 & 0,507 & $\underline{0,004^{* *}}$ & 24 & 0,194 & 0,364 & 35 & 0,257 & 0,136 & 33 & 0,465 & $\underline{0,006 * *}$ & 15 & $-0,352$ & 0,198 \\
\hline IDF & 31 & 0,160 & 0,390 & 24 & $-0,087$ & 0,684 & 35 & 0,248 & 0,150 & 33 & 0,472 & $\underline{0,006^{* *}}$ & 15 & 0,201 & 0,472 \\
\hline Conseg. Pac. & 31 & 0,469 & $\underline{0,008 * *}$ & 24 & 0,167 & 0,436 & 35 & 0,142 & 0,417 & 33 & 0,583 & $\leq 0,001^{* *}$ & 15 & $-0,163$ & 0,562 \\
\hline
\end{tabular}

FM: familiar mais importante para o paciente; fm: segundo familiar mais importante para o paciente; IAF: Índice de Acomodação Familiar; IDF: Índice de Desgaste

Familiar; Conseq. Pac.: consequência para o paciente da não participação do familiar na doença.

* Significativo a $5 \%$ de probabilidade pelo teste $t$ de Student.

** Significativo a $1 \%$ de probabilidade pelo teste $t$ de Student. 
Foram realizadas ainda correlações entre os índices da escala de acomodação familiar e entre outras variáveis, como intensidade global do TOC medido pela YBOCS e pela DYBOCS, intensidade de sintomas depressivos e ansiosos medidos pelas escalas de Beck e Hamilton, intensidade da falta de juízo crítico em relação aos $\mathrm{SOC}$ medido pela escala BABS, número de comorbidades e tempo de duração da doença (Tabela 5).

Tabela 5. Comparação entre índices de acomodação familiares e outras variáveis avaliadas nos pacientes com (TOC)

\begin{tabular}{|c|c|c|c|c|c|c|}
\hline & \multicolumn{2}{|c|}{ IAF } & \multicolumn{2}{|c|}{ IDF } & \multicolumn{2}{|c|}{ Conseq. Pac. } \\
\hline & $r$ & $\mathrm{p}$ & $r$ & $\mathrm{p}$ & $r$ & $\mathrm{p}$ \\
\hline $\begin{array}{l}\text { BDI } \\
(n=47)\end{array}$ & 0,326 & $0,025^{*}$ & 0,229 & 0,122 & 0,217 & 0,143 \\
\hline $\begin{array}{l}\text { BAl } \\
(n=47)\end{array}$ & 0,304 & $0,038^{*}$ & 0,006 & 0,970 & 0,172 & 0,247 \\
\hline $\begin{array}{l}\text { HAMA } \\
(\mathrm{n}=47)\end{array}$ & 0,473 & $0,001^{* *}$ & 0,124 & 0,407 & 0,225 & 0,128 \\
\hline $\begin{array}{l}\text { HAMD } \\
(n=47)\end{array}$ & 0,441 & $0,002^{* *}$ & 0,308 & $0,035^{*}$ & 0,338 & $0,020^{*}$ \\
\hline $\begin{array}{l}\text { YBOCS } \\
(n=45)\end{array}$ & 0,709 & $<0,001^{* *}$ & 0,500 & $<0,001^{* *}$ & 0,645 & $<0,001^{* *}$ \\
\hline $\begin{array}{l}\text { DYBOCS } \\
(\mathrm{n}=47)\end{array}$ & 0,641 & $<0,001^{* *}$ & 0,479 & $<0,001^{* *}$ & 0,532 & $<0,001^{* *}$ \\
\hline $\begin{array}{l}\text { BABS } \\
(n=45)\end{array}$ & 0,195 & 0,199 & 0,245 & 0,105 & 0,348 & $0,019^{*}$ \\
\hline $\begin{array}{l}\text { NUMCOMOR. } \\
(\mathrm{n}=47)\end{array}$ & 0,203 & 0,170 & 0,131 & 0,380 & 0,023 & 0,880 \\
\hline $\begin{array}{l}\text { DURASINT. } \\
(n=45)\end{array}$ & $-0,013$ & 0,931 & $-0,223$ & 0,140 & 0,065 & 0,672 \\
\hline
\end{tabular}

IAF: Índice de Acomodação Familiar; IDF: Índice de Desgaste Familiar; Conseq. Pac.: consequência para o paciente da não participação do familiar na doença; BDI: Beck Depression Inventory; BAI: Beck Anxiety Inventory; HAMA: Escala de Ansiedade de Hamilton; HAMD: Escala de Depressão de Hamilton; YBOCS: Yale-Brown Obsessive-Compulsive Scale; DYBOCS: Dimensional Yale-Brown Obsessive-Compulsive Scale; BABS: Brown Assessment of Beliefs Scale; NUMCOMOR.: número de comorbidades; DURASINT.: tempo de duração dos sintomas obsessivo-compulsivos.

* Significativo a $5 \%$ de probabilidade pelo teste $t$ de Student.

** Significativo a $1 \%$ de probabilidade pelo teste $t$ de Student.

O Índice de Acomodação Familiar mostrou correlações diretas moderadas com as intensidades dos sintomas ansiosos e depressivos medidas tanto pela escala de Beck quanto de Hamilton. Da mesma forma, mostrou-se diretamente correlacionada com a intensidade dos $\mathrm{SOC}$ medida tanto pela YBOCS quanto pela DYBOCS, mas nesse caso o nível de intensidade dessas correlações foi forte.

O Índice de Desgaste Familiar, por sua vez, mostrou correlação direta fraca com a HAMD e correlação direta moderada com a intensidade dos SOC medida tanto pela YBOCS quanto pela DYBOCS.

O Índice de consequências ao paciente devido à recusa do familiar em participar dos SOC mostrou correlação direta forte com a intensidade dos SOC, quando medida pela YBOCS, e moderada, quando medida pela DYBOCS. Mostrou ainda correlação direta fraca com a intensidade do juízo crítico medida pela BABS.

\section{DISCUSSÃO}

Apesar de não possuir grupo-controle e do pequeno tamanho amostral, este estudo evidencia aspectos clínicos importantes em relação ao funcionamento familiar de pacientes com TOC, utilizando pela primeira vez um instrumento (DYBOCS) que permite quantificar a intensidade de cada dimensão de sintomas obsessivo-compulsivos, possibilitando avaliar o quanto esses aspectos intrínsecos da fenomenologia descritiva do TOC influenciam no comportamento familiar.

Nossa amostra como um todo, contudo, pode ser comparável à literatura, pois os pacientes apresentaram média moderada na intensidade dos $\mathrm{SOC}$ e leve intensidade dos sintomas ansiosos e depressivos ${ }^{3,4,12,13}$. A possível morbidade psiquiátrica dos familiares considerados como principais cuidadores (FM) e cuidadores secundários (fm) precisa ser considerada em estudos futuros.

\section{Acomodação familiar e dimensões da DYBOCS}

Em relação às correlações entre $\mathrm{SOC}$ predominantes medidos pela DYBOCS e Índices de Acomodação Familiar, não se encontraram diferenças significativas entre agrupamentos sintomáticos e pior ou melhor acomodação familiar. Poderse-ia supor, por exemplo, que pacientes com maiores escores nas dimensões simetria e contaminação/limpeza produzissem maior diferença nos Índices de Acomodação Familiar, o que não se evidenciou neste estudo. Isso talvez tenha ocorrido em virtude do tamanho amostral pequeno, uma vez que houve tendência estatística dos pacientes com a dimensão contaminação e limpeza a apresentarem maiores índices de acomodação em relação aos que não possuíam tal dimensão, como encontrou Guedes ${ }^{8}$.

Por outro lado, segundo esta mesma autora, possivelmente não seja o conteúdo do SOC o fator que interfira no funcionamento familiar, mas sim o quão intrusivos se tornam os rituais e suas consequências ${ }^{21}$. Ou seja, o que importa em termos familiares é a gravidade da sintomatologia e o pouco juízo crítico do paciente acerca de seus sintomas, e não necessariamente o conteúdo do SOC.

\section{Criticismo percebido e DYBOCS}

Contrastando com outras doenças psiquiátricas (como esquizofrenia e transtornos do humor), os estudos dos fatores familiares envolvidos no TOC e a sua inter-relação com a doença ainda são bastante escassos. Com a aplicação da Escala de Criticismo Percebido aos pacientes, este trabalho encontrou informações interessantes sobre a percepção dos pacientes em relação aos seus familiares.

Pacientes com SOC de conteúdo agressivo, em média, valorizaram mais o suporte emocional oferecido pelo fm do que os pacientes sem SOC de conteúdo agressivo. Significa dizer que, quando há conteúdo agressivo envolvido, esses pacientes percebem-se mais apoiados pelos familiares de importân- 
cia secundária. Poder-se-ia especular que o conteúdo agressivo dos SOC estaria envolvendo exatamente os FM, tornando mais difícil a aproximação com esses familiares. Assinalaram, entretanto, julgar mais importante o auxílio proporcionado pelos familiares considerados mais importantes (FM), em comparação aos pacientes com outras dimensões do TOC.

Tais achados devem ser analisados levando-se em consideração que a dimensão agressiva do TOC é basicamente restrita a obsessões. Para evitar ações diretamente relacionadas a esses pensamentos, há produção de grande nível de ansiedade. Por meio de atos compensatórios, esses pacientes realizam rituais compulsivos desvinculados do conteúdo obsessivo, considerado absurdo pelo próprio paciente. Provavelmente, em comparação às outras dimensões avaliadas, a agressiva apresentase mais profundamente egodistônica e produtora de culpa.

Pode ser visto, ainda, na tabela 3, que pacientes colecionistas comumente consideram-se mais críticos e também mais suscetíveis a críticas dos fm. Interessante observar que esses resultados não foram vistos em relação aos FM. Isso pode ser consequência de um pior nível de juízo crítico dos pacientes sobre sua situação, o que os torna mais egossintônicos. Além disso, apresentam sintomas mais facilmente aceitos pelos próprios familiares mais próximos (FM) como algo "inofensivo". Quando familiares menos próximos (fm) e, portanto, não tão acomodados aos sintomas do paciente, criticam-no, ele pode exibir reações mais exacerbadas, até mesmo porque está sendo alvo de críticas a hábitos com os quais os familiares mais próximos conseguem muitas vezes conviver, tolerar ou até reforçar.

\section{Correlação das intensidades do funcionamento familiar com a DYBOCS}

A análise das correlações entre os escores de gravidade da DYBOCS e os escores de criticismo percebido e acomodação familiar mostrou correlações estatisticamente significativas e de moderada intensidade entre portadores da dimensão contaminação e limpeza. Quanto mais grave essa dimensão da DYBOCS, mais criticados e mais sensíveis às críticas pelos FM os pacientes se sentem. Também se sentem menos apoiados pelos fm em termos de suporte emocional. Esses resultados de criticismo percebido estão de acordo com a constatação clínica de que provavelmente o paciente com essa dimensão sinta-se mais facilmente criticado, pois acredita que seus pensamentos e atos sejam justificáveis, ou seja, possui menor capacidade crítica em relação aos seus sintomas. Houve correlações diretas moderadas para essa dimensão com os Índices de Acomodação Familiar. Limpeza e higiene são atos socialmente aceitos e, embora exagerados nos pacientes com TOC, a família se acomoda mais facilmente, o que acaba contribuindo para o agravamento da condição e maior intolerância do paciente a críticas, quando essas são feitas.

Para os pacientes com conteúdo agressivo, deve-se considerar a ocorrência de fenômenos indiretos. Como essa dimensão é predominantemente obsessiva, possui compulsões híbridas com outras dimensões. Por exemplo, a obsessão, apesar de possuir conteúdo agressivo, pode gerar compulsões de limpeza/lavagem, simetria, ordem, arranjo, contagem, religioso (rezar) ou compulsões mentais. Outra possível explicação é a culpa gerada por essas obsessões, ocasionando consequentemente maior intensidade de sintomas ansiosos e depressivos, que poderiam produzir acomodação familiar mais facilmente.

Lee et al. ${ }^{22}$, comparando 47 pacientes com TOC com 41 sujeitos da comunidade, encontraram que o perfeccionismo foi um fator associado a pior resposta ao tratamento. Apesar de não ser critério para TOC pelo DSM-IV, mas sim para transtorno de personalidade obsessivo-compulsiva², encontraram maiores índices de perfeccionismo em pacientes com TOC do que numa amostra populacional, utilizando a Escala Multidimensional de Perfeccionismo de Frost. Já o criticismo percebido teria sido fator de bom prognóstico ao tratamento com fluoxetina e terapia cognitivo-comportamental ${ }^{22}$.

\section{Controle de variáveis de confusão}

A associação de transtornos depressivos e ansiosos em comorbidade com o TOC é complicação bastante comum. Os transtornos depressivos ao longo da vida podem ser descritos em até $85 \%$ dos pacientes com TOC 9 . Em geral, o transtorno tem um impacto importante no funcionamento da vida diária e, com frequência, torna os pacientes socialmente isolados, com dificuldades de interação social e com alto índice de celibato ${ }^{23}$. Quase sempre ocorrem grandes dificuldades nos ambientes de trabalho, escola e família, provocadas pela inadequação social e afetiva que acompanha o TOC. Provavelmente, os transtornos depressivos devam-se, em grande parte, aos prejuízos causados pelo TOC, ou seja, ele acaba originando, ou mesmo agravando, um transtorno de humor preexistente. O mesmo ocorre com sintomas ansiosos, na medida em que são fenômenos inerentes à doença e ligados à regulação da tensão emocional produzida pelas obsessões ou pela não realização das compulsões.

Nesta amostra encontrou-se presença de correlação entre intensidade de sintomas depressivos e ansiosos e os Índices de Acomodação Familiar. Houve correlações diretas moderadas entre as medidas da HAMA e BAI com a acomodação familiar. Houve ainda diferença entre as correlações realizadas com a escala de depressão de Hamilton e de Beck. Maiores escores HAMD apresentaram correlações mais fortes com os Índices de Acomodação Familiar do que os escores medidos pela BDI. Pode-se deduzir apenas que a característica inerente a cada escala possa ter produzido essas diferenças: HAMD avaliando mais sintomas depressivos neurovegetativos e BDI avaliando melhor sintomas cognitivos. Pode-se, pois, inferir a partir disso que pacientes com TOC e sintomas depressivos com características mais "somáticas" teriam tendência a produzir maior acomodação familiar, des- 
gaste familiar e reações inapropriadas quando os familiares não participam de seus sintomas.

Neste estudo, encontrou-se também forte correlação direta entre a intensidade dos SOC medidos pela YBOCS e pela DYBOCS e o grau de acomodação familiar. Outros estudos registraram correlações semelhantes entre maior gravidade do TOC e maior acomodação familiar em adultos ${ }^{13,24,25}$. Storch et al. ${ }^{26}$ também encontraram essa correlação em uma amostra de familiares de 57 crianças e adolescentes entre 7 e 17 anos $^{26}$. Essas associações são importantes, sobretudo em termos terapêuticos, pois deve sempre ser levada em consideração a dinâmica familiar envolvida para que se obtenha maior chance de sucesso no tratamento do TOC ${ }^{10,21}$.

Encontrou-se associação positiva com maior acomodação familiar (particularmente no item da escala referente às reações negativas do paciente quando a família não reforçava os sintomas) com o nível de juízo crítico medido pela BABS, ou seja, quanto menor o juízo crítico do paciente em relação aos seus sintomas, maior será a reação negativa do paciente devida à recusa do familiar em assisti-lo nos seus sintomas. Ideias supervalorizadas ou juízo crítico pobre são tidos como preditores de pior resposta a tratamentos ${ }^{6}$. Assim é que um círculo vicioso pode ocorrer: com juízo crítico pobre o paciente opõe-se a ser tratado ou ajudado pelos familiares; sem tratamento/ajuda, os sintomas e suas consequências ficam mais graves; maior gravidade dos SOC e de suas consequências geram maior acomodação familiar, e assim por diante.

\section{CONCLUSÃO}

Instrumentos já consagrados como a YBOCS e, mais recentemente, a DYBOCS, fornecem perfis bastante confiáveis acerca das características mórbidas. No entanto, essas escalas não abordam atitudes familiares envolvidas na doença. Percebe-se, por isso, a necessidade de associação de instrumentos clínicos que mensurem confiavelmente gravidade e classe de sintomas com instrumentos de avaliação familiar, no sentido de compreender mais profundamente as várias facetas de uma mesma doença, correlacionando características de sintomas (dimensões) com as suas repercussões no ambiente familiar.

Subtipos específicos de doença obsessivo-compulsiva, bem como diferentes níveis de gravidade, podem ser influenciados pela maneira com que a família lida com o paciente. Não se pode, entretanto, em virtude da metodologia do estudo, definir se as alterações familiares são consequência ou causa dos diferentes padrões de doença dos pacientes (causalidade reversa). Deve-se considerar, ainda, que proporção considerável de familiares também tende a ser portadora, em situação plena ou de forma subclínica, dessa mesma condição. Esse é um fator que deverá ser avaliado em estudos futuros, para se poder estabelecer mais profundamente o amoldamento da família e a influência dela na doença do paciente. Ainda que a influência biológica seja importante caso se considere a predisposição genética herdada para a doença, já confirmada em diversos estudos ${ }^{27,28}$, a influência ambiental possui papel na manutenção dos sintomas ${ }^{13,29}$.

Em suma, os achados deste estudo fortalecem as informações já constatadas na prática clínica (mas ainda pouco estudadas) no que se refere ao importante papel desempenhado pela família do paciente com TOC. Escalas de avaliação do ajustamento familiar e da percepção crítica do paciente são instrumentos válidos que se incorporam ao vasto arsenal de pesquisa sobre o TOC, pois propiciam uma estimativa quantitativa que auxilia na formulação de intervenções terapêuticas mais efetivas.

\section{REFERÊNCIAS}

1. Leckman JF, Grice DE, Boardman J, Zhang H, Vitale A, Bondi C, et al. Symptoms of obsessive-compulsive disorder. Am J Psychiatry. 1997;154:911-7.

2. American Psychiatry Association. Diagnostic and Statistical Manual for Psychiatry Disorders. 4. ed. Washington: American Psychiatry Press; 1994.

3. Goodman WK, Price LH, Rasmussen S, Mazure C, Fleischmann RL, Hill CL, et al. The YaleBrown Obsessive-Compulsive Scale: development, use and reliability. Arch Gen Psychiatry. 1989;46:1006-11.

4. Goodman WK, Price LH, Rasmussen S, Mazure C, Delgado P, Heninger GR, et al. The YaleBrown Obsessive-Compulsive Scale: validity. Arch Gen Psychiatry. 1989;46:1012-6.

5. Stekette G. Disability and family burden in obsessive-compulsive disorder. Can J Psychiatry. 1997;42(9):919-28.

6. Geffken GR, Storch EA, Duke DC, Monaco L, Lewin AB, Goodman WK. Hope and coping in family members of patients with obsessive-compulsive disorder. J Anxiety Disord. 2006;20(5):614-29.

7. Albert U, Salvi V, Saracco P, Bogetto F, Maina G. Health-related quality of life among firstdegree relatives of patients with obsessive-compulsive disorder in Italy. Psychiatr Serv. 2007:58(7):970-6.

8. Guedes ML. Transtorno obsessivo-compulsivo: um estudo do processo de acomodação familiar [dissertação]. São Paulo: Escola Paulista de Medicina, Universidade Federal de São Paul0; 1997. p. 80.

9. Ferrão YA. Características clínicas do transtorno obsessivo-compulsivo refratário aos tratamentos convencionais [tese]. São Paulo: Universidade de São Paulo; 2004. p. 137.

10. Steketee $G$, Van Noppen B. Family approaches to treatment for obsessive compulsive disorder. Rev Bras Psiquiatr. 2003;25(1):43-50.

11. Baer $L$. Factor analysis of symptom subtypes of obsessive compulsive disorder and their relation to personality and tic disorders. J Clin Psychiatry. 1994;55(March suppl):18-23.

12. Rosario-Campos MC, Miguel EC, Quatrano S, Chacon P, Ferrao Y, Findley D, et al. The Dimensional Yale-Brown Obsessive-Compulsive Scale (DY-BOCS): an instrument for assessing obsessive-compulsive symptom dimensions. Mol Psychiatry. 2006;11(5):495-504.

13. Ferrao YA, Shavitt RG, Bedin NR, De Mathis ME, Lopes AC, Fontenelle LF, et al. Clinical features associated to refractory obsessive-compulsive disorder. J Affect Disord. 2006;94(1-3):199-209.

14. First MB, Spitzer RL, Gibbon M, Williams JBW. Structured Clinical Interview for DSM-IV axis I disorders: clinical version (SCID-CV). Washington, DC: American Psychiatric Press; 1997.

15. Eisen JL, Phillips KA, Baer L, Beer DA, Atala KD, Rasmussen SA. The Brown Assessment of Beliefs Scale: reliability and validity. Am J Psychiatry. 1998;155:102-8.

16. Beck AT, Ward CH, Mendelson M, Mock J, Erbaugh J. An inventory for measuring depression. Arch Gen Psychiatry. 1961;12(4):561-71.

17. Beck AT, Epstein N, Brown G, Steer RA. An inventory for measuring clinical anxiety: psychometric properties. J Consult Clin Psychol. 1988;56(6):893-7.

18. Hamilton M. Development of a rating scale for primary depressive illness. Br J Soc Clin Psychol. 1967;6(4):278-96. 
19. Hamilton M. The assessment of anxiety states by rating. Br J Med Psychol. 1959;32:50-5.

20. Calvocoressi L, Lewis B, Harris M, Trufan S, Goodman W, Mcdougle C, et al. Family accommodation in obsessive-compulsive disorder. Am J Psychiatry. 1995;152(3):441-3.

21. Guedes ML. Relação família-paciente no transtorno obsessivo-compulsivo. Rev Bras Psiquiatr. 2001;23(Supl II):65-7.

22. Lee JC, Prado HS, Rosário MC, Diniz JB, Borcato S, Belotto $C B$, et al. Perfectionism and sensory phenomena as important predictors of treatment response in patients with obsessive-compulsive disorder. Compr Psychiatry. 2009;50(5):431-6.

23. Hollander E, Simeon D. Transtornos de ansiedade. 1. ed. Porto Alegre: Artmed; 2004.

24. Calvocoressi L, Mazure C, KasI S, Skolnick J, Fisk D, Vegso S, et al. Family accommodation of obsessive-compulsive symptoms: instrument development and assessment of family behavior. J Nerv Ment Dis. 1999;187(10):636-42.
25. Amir N, Freshman M, Foa EB. Family distress and involvement in relatives of obsessivecompulsive disorder patients. J Anxiety Disord. 2000;14(3):209-17.

26. Storch EA, Geffken GR, Merlo LJ, Jacob ML, Murphy TK, Goodman WK, et al. Family accommodation in pediatric obsessive-compulsive disorder. J Clin Child Adolesc Psychol. 2007;36(2):207-16

27. Nestadt G, Samuels J, Riddle M, Bienvenu J, Liang K, LaBuda M, et al. A family study of obsessive-compulsive disorder. Arch Gen Psychiatry. 2000;57:358-63.

28. Lenane M, Swedo S, Leonard H, Pauls D, Sceery W, Rapoport J. Psychiatric disorders in first degree relatives of children and adolescents with obsessive-compulsive disorder. J Am Acad Child Adolesc Psychiatry. 1990;29:407-12.

29. Ferrão Y, Aguiar P, Minuzzi L, Grillo R, Lopes R, Rosa R. Características clínicas e história familiar em pacientes ambulatoriais com transtorno obsessivo-compulsivo. Rev Psiquiatr RS. 2004;26 (3):274-9. 